\title{
Coulomb blockade phenomena observed in supported metallic nanoislands
}

\author{
I-Po Hong 1,2, Christophe Brun ${ }^{1,3}$, Marina Pivetta ${ }^{1}$, François Patthey ${ }^{1}$ and Wolf-Dieter Schneider ${ }^{1,4 *}$ \\ ' Faculté des Sciences de Base, Ecole Polytechnique Fédérale de Lausanne, Institut de Physique de la Matière Condensée, Lausanne, Switzerland \\ ${ }^{2}$ Key Laboratory for Physics and Chemistry of Nanodevices and Department of Electronics, Peking University, Beijing, China \\ ${ }^{3}$ Institut des Nanosciences de Paris, Université Pierre et Marie Curie and CNRS-UMR 7588, Paris, France \\ ${ }^{4}$ Department of Chemical Physics, Fritz-Haber-Institute of the Max-Planck-Society, Berlin, Germany
}

\section{Edited by:}

Peter Fischer, Lawrence Berkeley

National Laboratory, USA

Reviewed by:

Aidan Hindmarch, Durham

University, UK

Shuji Hasegawa, University of

Tokyo, Japan

Sebastian Loth, Max Planck Institute for Solid State Research, Germany

Richard Berndt, IEAP, CAU Kiel,

Germany

*Correspondence:

Wolf-Dieter Schneider, Ecole

Polytechnique Fédérale de

Lausanne, Institut de Physique de la

Matière Condensée, Cubotron 421,

CH-1015 Lausanne, Switzerland

e-mail:wolf-dieter.schneider@epfl.ch
The electron transport properties of single crystalline metallic nanostructures in the Coulomb blockade (CB) regime have been investigated by low-temperature scanning tunneling spectroscopy. To this end, nanoscale flat-top $\mathrm{Pb}$ islands with well-defined geometries are grown on $\mathrm{NaCl}$-covered $\mathrm{Ag}(111)$ substrates. The tunneling spectra acquired at $4.6 \mathrm{~K}$ on the $\mathrm{Pb}$ nanoislands reflect the presence of single electron tunneling processes across the double-barrier tunnel junction (DBTJ). By a controlled change of the tip-island tunnel distance, the spectra display the characteristic evolution from CB to Coulomb staircase (CS) regime. Simulations within the semi-classical orthodox theory allow us to extract quantitatively the parameters characterizing the DBTJ, i.e., the resistances, capacitances, and the residual charge $Q_{0}$. Manipulation of $Q_{0}$ is achieved by controlled application of voltage pulses on the $\mathrm{Pb}$ islands. Moreover, under specific tunneling conditions, the influence of the tip-island junction on $Q_{0}$ is revealed in topographic images of the $\mathrm{Pb}$ islands.

Keywords: coulomb blockade, STM, STS, Pb-nanoislands, dielectric support

\section{INTRODUCTION}

The study of Coulomb blockade (CB) phenomena in nanostructures is a very attractive area of condensed matter physics, because single electron electronics is considered to have a high potential for basic research and for technological applications $(1-5)$. The field started more than 40 years ago with the investigation of small metal particles embedded in an oxide layer between two planar metal electrodes $(6,7)$. Subsequently, in order to avoid ensemble effects and to study single doublebarrier tunnel junctions (DBTJ), CB phenomena have been investigated in small-area tunnel junctions prepared by lithographic methods developed for microelectronics $(8,9)$. More recently, junctions using a scanning tunneling microscope (STM) tip as one adjustable electrode came into the focus of research (10-13).

Consider single electron transport in the system shown in Figure 1: the two barriers constitute a DBTJ. For each junction the barriers are modeled by an ohmic resistor $R_{i}$ in parallel with a capacitor $C_{i}, i=1$ for the substrate-island junction and $i=2$ for the island-tip junction, as shown in the equivalent electronic circuit in Figure 1B. In order to transfer a single electron through the DBTJ, the electron must overcome the Coulomb charging energy $E_{c}=e^{2} / C_{\Sigma}$, where $C_{\Sigma}=\sum C_{i}$. If the charging energy $E_{c}$ is much larger than the thermal energy $k_{B} T$, the tunneling of an electron is blocked for bias voltages smaller than $U_{c}=e / C_{\Sigma}$. This phenomenon has been termed CB (CB) $(2,3,14-16)$. Moreover, in order to keep charge fluctuations sufficiently small, the coupling resistance of each junction has to be significantly larger than the quantum resistance: $R_{i} \gg R_{Q}=h /\left(2 e^{2}\right) \approx 12.9 \mathrm{k} \Omega$. These conditions imply that extremely small metal clusters have to be probed, or that the measurements have to be performed at low temperature.

Previous studies of nanostructures in the CB regime by STM were performed on amorphous particles $(11,13,17,18)$, on particles covered by shells consisting of specific ligands and/or molecules (19-22), and to a lesser extent on supported clusters and metal islands $(18,23-26)$. The vast majority of the $\mathrm{CB}$ spectroscopy results have been successfully described within the orthodox theory of the DBTJ (2), which allowed the authors to determine quantitatively the tip-particle and the particlesubstrate junction parameters (see Figure 1B). Within the context of single electron tunneling, the "orthodox theory," a semiclassical theory, is based on the following three assumptions (3). (1) The electron energy quantization inside the conductors is ignored, i.e., the electron energy spectrum is continuous. (2) Coherent quantum processes consisting of several simultaneous tunneling events ("cotunneling") are ignored. This assumption is valid if the resistance of all tunneling barriers of the system is much higher than the quantum unit of resistance. Only under this condition the quantum-mechanical uncertainty of electrons is suppressed because of the high tunneling resistance (i.e., less tunneling events). Thus one can neglect the concurrent tunneling events and treat one electron at a time, which makes controllable single-electron manipulation possible. (3) The tunneling time $\tau_{t}$ of an electron tunneling through the barrier is assumed to be negligible compared to other time scales (including the interval between subsequent tunneling events). For a typical tunneling event in a practical junction $(3), \tau_{t}$ is around $10^{-15} \mathrm{~s}$. For 


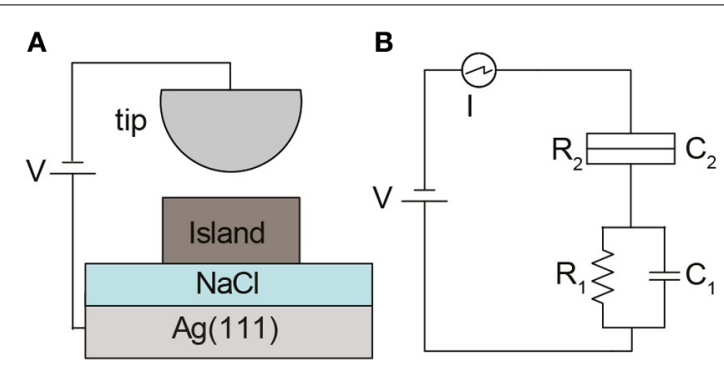

FIGURE 1 | (A) Schematic diagram of the Coulomb blockade system. The $\mathrm{Pb}$ nanoisland is embedded between the vacuum barrier and a few $\mathrm{NaCl}$ dielectric layers on $\mathrm{Ag}(111)$. (B) Equivalent double-barrier tunnel junction (DBTJ) circuit where each junction is represented by a set of capacitors and resistors, connected in parallel.

a typical STM tunneling junction with $1 \mathrm{nA}$ tunneling current, on average one electron tunnels per $10^{-10} \mathrm{~s}$. Considering that the electronic lifetime of an electron in the states of the islands is around $10^{-13}-10^{-15} \mathrm{~s}$, electrons tunnel one at a time and consequently under these conditions cotunneling processes can be neglected.

Recently, in a low-temperature scanning tunneling spectroscopy (STS) study, we reported (27) on the observation of the dynamical Coulomb blockade (DCB) (28-30) in nanosized electrical contacts consisting of small $\mathrm{Pb}$ islands on various conducting, semiconducting, and partially insulating substrates. We observed a suppression of the differential tunnel conductance at small bias voltages due to DCB effects. The differential conductance spectra allowed us to determine the capacitances and resistances of the electrical contact which depend systematically on the island-substrate contact area. Calculations based on the theory of environmentally assisted tunneling agree well with the measurements.

Here, we extend the previous observations from the DCB to the orthodox $\mathrm{CB}$ and Coulomb staircase (CS) regime. We report on the observation of $\mathrm{CB}$ phenomena in $\mathrm{Pb}$ nanoislands, ranging from a lateral size of a few nanometers to tens of nanometers and a thickness of a few monolayers, grown on ultrathin dielectric films of $\mathrm{NaCl}$ deposited on $\mathrm{Ag}(111)$. For selected $\mathrm{Pb}$ islands of well-defined area, the local tunneling spectra reflect CB and CS phenomena in the DBTJ (see Figure 1) characteristic for single electron tunneling: Every additional electron tunneling to or from the $\mathrm{Pb}$ nanoisland is counted one by one. Using a model based on the orthodox theory of single electron tunneling $(12,16)$, we are able to simulate the experimental tunneling spectra and to extract the relevant parameters of the DBTJ, i.e., the resistances of islandsubstrate and tip-island junctions $\left(R_{1}\right.$ and $\left.R_{2}\right)$, the capacitances of island-substrate and tip-island junctions $\left(C_{1}\right.$ and $\left.C_{2}\right)$, and the residual charge $\left(Q_{0}\right)$ on the island. The extracted $C_{1}$ capacitances fit well with the values corresponding to a planar capacitor, $C_{1}=\varepsilon_{0} \varepsilon_{r} A_{\text {island }} / d$, the island area $A_{\text {island }}$ being measured from the STM topography and the insulator thickness determined by field emission resonances (FERs) analysis. Manipulation of $Q_{0}$ is achieved by controlled application of voltage pulses on the $\mathrm{Pb}$ islands. Moreover, under specific tunneling conditions, the influence of the adjustable tip-island junction on $Q_{0}$ is revealed in topographic images of the $\mathrm{Pb}$ islands.

In an STM measurement of a DBTJ, in general the tip-island junction resistance $R_{2}$ is always much larger than the quantum resistance $R_{Q}$. Then the electron transport through such a DBTJ can be separated into three different regimes (16): if the islandsubstrate resistance $R_{1} \ll R_{Q}$, the whole system corresponds to a single junction; if $R_{1}$ is of the order of $R_{Q}$, the dynamical Coulomb blockade (DCB) regime is reached; if $R_{1} \gg R_{Q}$, the $\mathrm{CB}$ and CS regime is entered. These different conditions can be selectively and controllably fulfilled in our experimental setup by choosing the appropriate size of the islands (by changing the growth parameters) and the pertinent tunnel parameters, including an adjustable tip-island distance (by changing the tip-island resistance $R_{2}$ ). In this way island-size-dependent CB gaps and CS have been unambiguously identified in the tunneling spectra obtained on the $\mathrm{Pb}$ quantum dots on $\mathrm{NaCl} / \mathrm{Ag}(111)$.

\section{EXPERIMENTAL}

The $\mathrm{Ag}(111)$ single crystal substrate was cleaned by $\mathrm{Ar}^{+}$sputtering and subsequent annealing cycles. $\mathrm{NaCl}$ powder was thermally evaporated from a crucible at a temperature of $T=620^{\circ} \mathrm{C}$ onto the Ag substrate held at room temperature or heated to $\approx 420 \mathrm{~K}$. Subsequently, $\mathrm{Pb}$ islands were grown by evaporation of $\mathrm{Pb}$ from a W filament onto the $\mathrm{NaCl}$-covered Ag substrate cooled to $130 \mathrm{~K}$ to obtain $\mathrm{Pb}$ islands of the desired size.

The experiments were conducted in a homebuilt STM operated at $4.6 \mathrm{~K}(31)$. Differential conductance $(d I / d V)$ measurements were performed with an open feedback loop using a lock-in technique with modulation voltage from 2 to $10 \mathrm{mV}_{\mathrm{pp}}$ at 300 $400 \mathrm{~Hz}$ frequency with tunneling current ranging from $100 \mathrm{pA}$ to a few $n A$.

\section{RESULTS AND DISCUSSION}

\subsection{GROWTH OF Pb NANOISLANDS ON NaCL/Ag(111)}

A bulk $\mathrm{NaCl}$ crystal is a dielectric material with a wide band gap in the range of $8.5-9 \mathrm{eV}(32-34)$. For thin $\mathrm{NaCl}$ films, the band gap is reported to be close to the bulk value (35) For $1-3 \mathrm{ML}$ of $\mathrm{NaCl}$ on $\mathrm{Ag}(100)$, recent STS measurements of the energy positions of field emission resonances propose a work function of $3.2 \mathrm{eV}$ (36), while ultraviolet photoemission spectroscopy (UPS) yields $3.5 \mathrm{eV}(36,37)$. From $d I / d V$ spectra measured on top of a $2 \mathrm{ML} \mathrm{NaCl}$ film, we deduce that the gap lies within the voltage range of $\pm 2 \mathrm{eV}$ around $E_{F}$, which is the energy range selected for the measurement of the $d I / d V$ spectra of the $\mathrm{Pb}$ nanoislands on $\mathrm{NaCl}$.

Studies of $\mathrm{NaCl}$ layers on other substrates abound, e.g., on $\operatorname{Al}(111)(35,38)$, on $\mathrm{Cu}(111)$ (39), on $\operatorname{Ag}(100)(36,40,41)$, and on $\mathrm{Au}(111)(42-44) . \mathrm{NaCl}(100)$ layers are very versatile as a dielectric ultrathin film for the electronic decoupling of supported molecules $(39,42,43)$ or other nanostructures (27) from a metallic substrate.

Here, $\mathrm{NaCl}$ was deposited on the substrate held at two different temperatures, $300 \mathrm{~K}$, and $\approx 420 \mathrm{~K}$, the latter favoring the formation of more extended, flatter, and defect-free layers. Figures $2 A, B$ show STM images corresponding to these two different sample preparations, yielding $\mathrm{Pb}$ islands of different average size. This 
behavior is mainly due to the higher density of nucleation points on the $\mathrm{NaCl}$ layer in (b) with respect to (a). The $\mathrm{NaCl}$ layer has different characteristics in the two cases: the layer is flat and presents very few defect in (a), while it shows the coexistence of various thicknesses and a higher density of edges in (b). In (a) the side length of the equilateral triangular $\mathrm{Pb}$ islands is in the range of 25 to $60 \mathrm{~nm}$, while in (b) it is $\leq 10 \mathrm{~nm}$. For the STS measurements, triangular islands of various sizes were selected. The $\mathrm{Pb}$ islands expose (111) crystal faces. A determination of the thickness of $\mathrm{NaCl}$ layers at a given location is not straightforward. Moreover, the apparent height of $\mathrm{NaCl}$ layers varies depending on tunneling conditions, such as bias voltage and tip conditions.

\subsection{COULOMB BLOCKADE AND COULOMB STAIRCASE ON Pb NANOISLANDS}

In a STM double junction geometry, there are some intrinsic limitations in the relations between resistances and capacitances of the two junctions. As discussed in the introduction, in order to observe $\mathrm{CB}$ phenomena, the condition $R_{i} \gg R_{Q}$ has to be satisfied. In the tunneling regime, this condition is easily satisfied for junction 2 (tip-vacuum-island), for which resistances $R_{2}$ of $1 \mathrm{M} \Omega$ to $1 \mathrm{G} \Omega$ are usual. For junction 1 , this condition requires the presence of an insulating layer of sufficient thickness, leading in our case to resistances $R_{1}$ of the order of $1 \mathrm{M} \Omega$ to $100 \mathrm{M} \Omega$. Concerning the capacitance, although the tip-island distance is of the same order as the insulating layer thickness $(0.5$
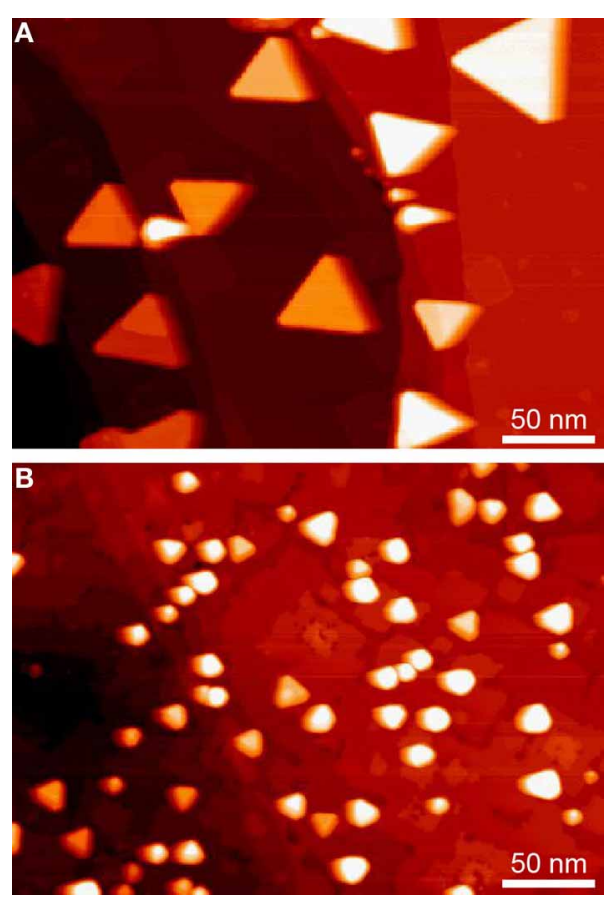

FIGURE 2 | STM topographies acquired on two different samples with $\mathbf{P b}$ islands grown on $\mathbf{N a C l} / \mathbf{A g}(\mathbf{1 1 1})$. $\mathrm{Pb}$ islands are visible in both images, most of them with triangular shape and a few with rounded shape. The smaller island size in (B) as compared to (A) is obtained on a $\mathrm{NaCl}$ film exhibiting more defects. (A): +1 V, $20 \mathrm{pA}$; (B): +4V, $20 \mathrm{pA}$. to $1 \mathrm{~nm}$ ), the $\mathrm{NaCl}$ dielectric constant plays an important role, leading to capacitances $C_{1}$ ten times larger than typical values for $C_{2}$. By varying the set-point current, the tip-island distance is modified and consequently also $R_{2}$ and $C_{2}$. The resistance, however, decreases exponentially with decreasing distance, while the capacitance increases only weakly.

Figure 3 shows $d I / d V$ spectra acquired on four different $\mathrm{Pb}$ islands. The spectra present the typical signature of $\mathrm{CB}$, i.e., a zero-conductance gap around the Fermi energy $\left(E_{\mathrm{F}}\right)$. Notice that bulk $\mathrm{Pb}$ is superconducting at the measurement temperature of $T=4.6 \mathrm{~K}$. We neglect here the possible existence of the superconducting gap in the $\mathrm{Pb}$ islands because the energy scale of the Coulomb gap is much larger than the one of the superconducting gap of $\leq 1 \mathrm{meV}$ (45). Consequently, we treat the tunneling junctions as if they are in the normal state. The width of the gap is inversely proportional to the island area $A_{\text {island: the gap }}$ is $\approx 120 \mathrm{mV}$ wide for the smallest island $\left(A_{\text {island }} \approx 20 \mathrm{~nm}^{2}\right)$, and $\approx 18 \mathrm{mV}$ for the largest one $\left(A_{\text {island }} \approx 220 \mathrm{~nm}^{2}\right)$. This is the expected behavior, since for $C_{2}<C_{1}$ the gap width is governed by the tip-substrate capacitance $C_{1}$. Therefore, as $C_{1}$ increases for increasing island area, it leads to narrower gaps. The $220 \mathrm{~nm}^{2}$ island (a triangle with $22 \mathrm{~nm}$ side) represents the upper limit for an unambiguous observation of a zero-conductance gap at this temperature. From the measured gap, we estimate $C_{1}$ to approximately $10 \mathrm{aF}$. For the $20 \mathrm{~nm}^{2}$-island, $C_{1}$ is ten times smaller. These values are in agreement with values calculated using the expression for a parallel plate capacitor $C_{1}=\varepsilon_{0} \varepsilon_{r} A_{\text {island }} / d_{\mathrm{NaCl}}$, with the island supported on a $3 \mathrm{ML} \mathrm{NaCl}$ film of thickness $d_{\mathrm{NaCl}}=$ $0.8 \mathrm{~nm}$ and dielectric constant of bulk $\mathrm{NaCl} \varepsilon_{r}=5.5$ (46) The small features in Figure 3A just below $\pm 0.1 \mathrm{eV}$ are related to the capacitance $C_{2}$ (see discussion of Figure 5).

Figure 4 displays the typical evolution of the tunneling spectra from the $\mathrm{CB}$ to the $\mathrm{CS}$ regime for a selected $\mathrm{Pb}$ triangular

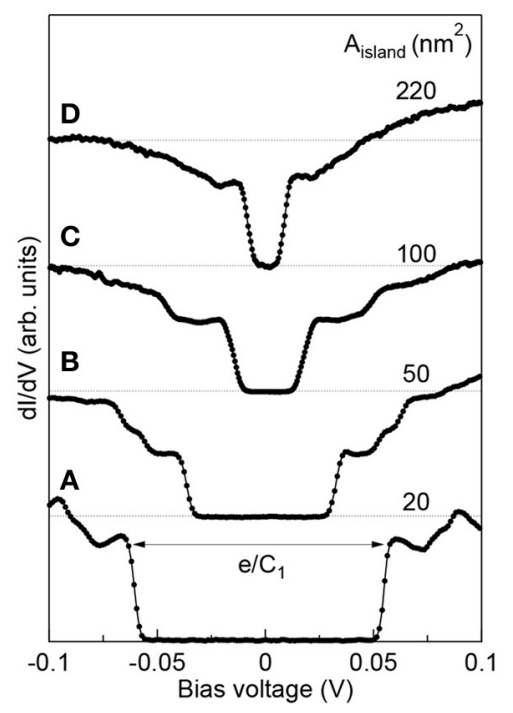

FIGURE 3 | dl/dV spectra labeled ( $A$ to $D$ ) acquired on four $\mathrm{Pb}$ islands with different area $\boldsymbol{A}_{\text {island. }}$. A Coulomb gap of decreasing size is visible in going from spectrum (A to $\mathbf{D}$ ) reflecting a Coulomb blockade process. 
island with a side length of $14 \mathrm{~nm}$. The $d I / d V$ spectrum A shows a simple Coulomb gap while spectra B to E display a series of equidistant peaks (steps in the $I-V$ characteristics, a CS) with increasing intensity. This progression is achieved by measuring

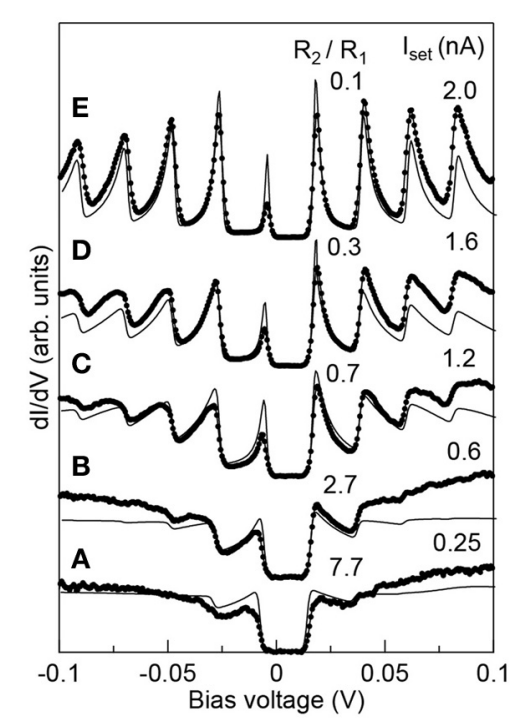

FIGURE 4 | Evolution from Coulomb blockade to Coulomb staircase: experimental STS data acquired on a $\mathrm{Pb}$ island with $14 \mathrm{~nm}$ side $\left(A_{\text {island }} \approx 85 \mathrm{~nm}^{2}\right)$ (dots) and simulation (black line) based on the orthodox theory. The spectra are offset for clarity. Parameters of the simulation: $R_{1}=39 \mathrm{M} \Omega, C_{1}=7.46 \mathrm{aF}$, and $C_{2}=0.7 \mathrm{aF} ;(\mathbf{A}): R_{2}=300 \mathrm{M} \Omega$ and $Q_{0}=-0.15 e,(B): R_{2}=105 \mathrm{M} \Omega$ and $Q_{0}=-0.2 e$, (C): $R_{2}=26 \mathrm{M} \Omega$ and $Q_{0}=-0.26 e$, (D): $R_{2}=13 \mathrm{M} \Omega$ and $Q_{0}=-0.26 e,(E): R_{2}=4.5 \mathrm{M} \Omega$ and $Q_{0}=-0.28 e$.

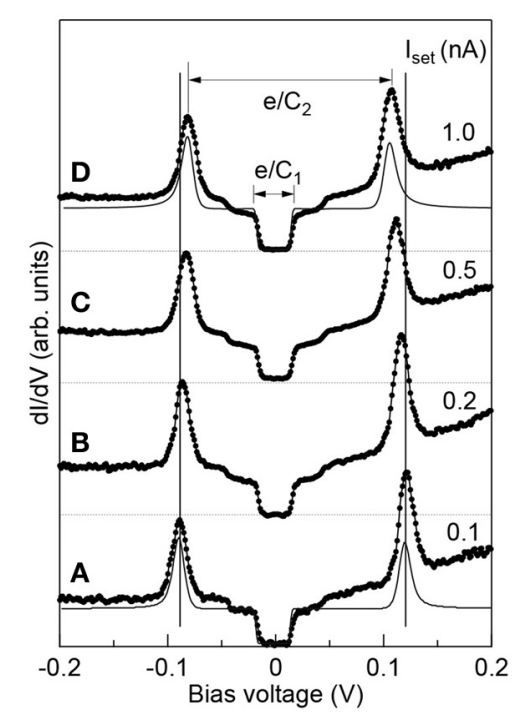

FIGURE 5 | Spectra (A to D): increase of the tip-island capacitance $C_{2}$ with decreasing tip-island distance (increasing tunnel current). The spectra, offset for clarity as $d l / d V(O V)=0$, have been acquired on a $\mathrm{Pb}$ island of $14 \mathrm{~nm}$ side. Parameters of the simulation, performed for spectra (A) and (D) curve: $C_{1}=5.0 \mathrm{aF}, C_{2}=0.77 \mathrm{aF}, R_{1}=5 \mathrm{M} \Omega, R_{2}=1700 \mathrm{M} \Omega$, $Q_{0}=0.062 \mathrm{e} ;$ (D) curve: $C_{1}=5.0 \mathrm{aF}, C_{2}=0.86 \mathrm{aF}, R_{1}=5 \mathrm{M} \Omega$, $R_{2}=145 \mathrm{M} \Omega, Q_{0}=0.07 e$. the tunnel spectra with increasing initial current, corresponding to decreasing tip sample separation and decreasing resistance $R_{2}$, in going from spectrum A to E. Our simulation within the orthodox theory reproduces the general features of the measured spectra revealing CB and CS characteristics. The simulation of these spectra within orthodox theory allows us to extract the pertinent parameters of the double barrier system, i.e., the capacitances and resistances of both junctions, as well as the residual non-integer charge $Q_{0}$ on the $\mathrm{Pb}$ island, see figure caption. Notice that, with decreasing tip-sample distance, $R_{2}$ decays exponentially, whereas $C_{2}$ increases linearly $(13,18)$. As this latter variation is small, $C_{2}$ is assumed to be constant throughout this series of simulations. To summarize, tunneling spectra on such nanoislands may display a simple CB gap or a CS depending on the parameters of the DBTJ. Well-developed CS's are observed with an asymmetric resistance ratio $R_{2}<R_{1}$.

Concerning the robustness of the simulations and the precision of the derived parameters we note that $Q_{0}$ is always determined with an error of $<10 \%$, as it corresponds to an energy shift of the tunneling spectra with respect to zero bias voltage. The capacitances $C_{1}$ and $C_{2}$ are also reasonably well determined, especially in situations like those depicted in Figure 5 or Figure 6 where two well separated energies are well-defined. The errors of the determined values are thus also $<10 \%$. The sum of $R_{1}$ and $R_{2}$ is fixed by the experimentally determined $I(V)$ characteristics. Their ratio is most favorably evaluated in the CS regime, as the height and the width of the various peaks are linked directly to their ratio. In this situation a precision of the order of $10 \%$ is obtained (see Figure 4). In the simple CB case, the precision of the resistance ratio is $\geq 10 \%$.

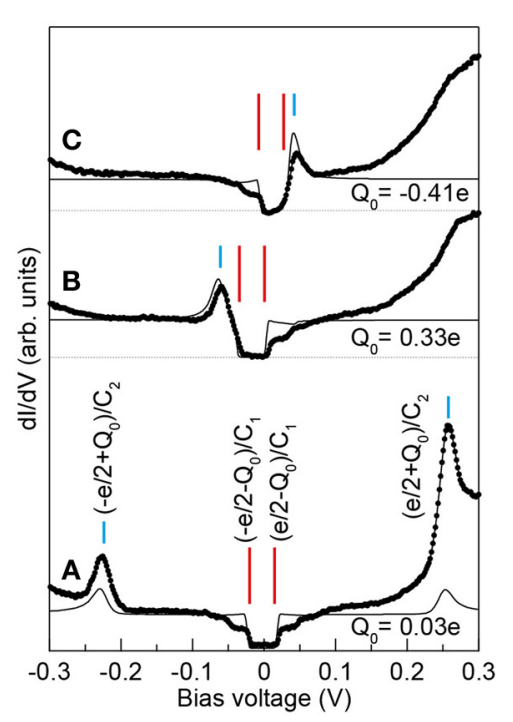

FIGURE 6 | Manipulation of the residual charge $\boldsymbol{Q}_{\mathbf{0}} . d l / d V$ spectra measured on the same island with $7.9 \mathrm{~nm}$ side, showing features related to both, $C_{1}$ and $C_{2}$, with varying $Q_{0}$, and corresponding simulations. The residual charge $\left(Q_{0}\right)$ of the island is changed by a bias voltage pulse (duration: $50 \mu \mathrm{s}$, current: $1 \mathrm{nA}$ ). Parameters of the simulation: $C_{1}=3.8 \mathrm{aF}$, $C_{2}=0.335 \mathrm{aF}, R_{1}=25 \mathrm{M} \Omega, R_{2}=2300 \mathrm{M} \Omega ;(\mathbf{A}): Q_{0}=0.03 \mathrm{e}$

(B): $Q_{0}=0.33 e,(\mathbf{C}): Q_{0}=-0.41 e$ 
A closer comparison of calculations and data in Figure 4 shows that there are some deviations in the intensity of the structures. For the present island of a thickness of $11 \mathrm{ML}$, a quantum well state, reflecting the vertical confinement, located at $+0.2 \mathrm{eV}$ above $E_{F}$ contributes a considerable background to the spectra (47). Therefore, a quantitative fitting of the tunnel spectra within the orthodox theory should consider the density of states (DOS) of the sample (48).

If the parameters of the DBTJ are appropriately chosen, spectral features reflecting both, tip-island $\left(C_{2}\right)$ and island-substrate $\left(C_{1}\right)$ capacitance can be observed simultaneously. Note that the electrostatic energy of the island is given by the Coulomb charging energy $E_{c}=e^{2} / C_{\Sigma}$, where $C_{\Sigma}=\sum C_{i}$. However, the threshold voltage necessary for the transfer of an electron across the tipisland (island-substrate) junction is inversely proportional to the capacitance $C_{2}\left(C_{1}\right)(12,13)$. Spectra of Figure 5 were acquired on a $\mathrm{Pb}$ island of $\approx 14 \mathrm{~nm}$ side length. Spectrum A shows a Coulomb gap around $E_{\mathrm{F}}$ as well as two peaks at $\approx \pm 0.1 \mathrm{~V}$, which are related to the $C_{2}$ capacitance. For decreasing tip-island distance (increasing tunnel current), the tip-island capacitance $C_{2}$ is expected to increase slightly. This behavior is observed in Figure 5B: by approaching the tip to the island from $A$ to $D$, the value of $C_{2}$ increases leading to a decreased separation between the two $C_{2}$ peaks, as indicated by the vertical lines. In addition, if the island area $A_{\text {island }}$ becomes comparable to the effective tip area $A_{\text {tip }}$, there will be also a contribution to $C_{2}$ due to the tipsubstrate capacitance. In the present measurement $A_{\text {island }}$ turns out to be smaller than $A_{\text {tip }}$. $C_{2}$ is then evaluated using the simple parallel-plate capacitance formula. In addition to the capacitance between the metallic island and the tip, the capacitance between the tip and the substrate, including the vacuum and the $\mathrm{NaCl}$ layers, is considered in the model.

The present measurements and their analysis allow us to obtain the dielectric constant of the ultrathin $\mathrm{NaCl}$ film within a parallel plate capacitor model, if we take the island substrate capacitance $C_{1}$ from the simulation of the spectra, the area $A_{\text {island }}$ of the capacitor plate from the STM topographies, and the thickness $d_{\mathrm{NaCl}}$ of the $\mathrm{NaCl}$ film as deduced from STS of FERs (36) With a typical island size of $A_{\text {island }}=100 \mathrm{~nm}^{2}$ and $d_{\mathrm{NaCl}}=$ $0.8 \mathrm{~nm}(3 \mathrm{ML})$, we obtain $\varepsilon_{r}=4$, a reasonable value compared to the one of $\varepsilon=5.5$ for bulk $\mathrm{NaCl}$ (46), and the one extracted from FERs measurements, $\varepsilon=3.5$ (36).

In contrast to the situation in a planar tunnel junction (49), our setup has no gate electrode. However, by externally applying a bias voltage pulse to the junction (12), a gate voltage change is mimicked. In Figure 6 we present three tunneling spectra, obtained on the same position on a triangular island of $7.9 \mathrm{~nm}$ side, where subsequently a bias voltage pulse of $\pm 5 \mathrm{~V}$ has been applied to the junction. The spectra clearly show the result of this action, a pulse-bias-dependent shift of the central Coulomb gap away from the symmetric zero-voltage position, accompanied by a shift of the $C_{2}$-related spectral features. The background above $+0.2 \mathrm{~V}$ originates from the tail of a quantum well state at higher energy (47). This background contributes also to the observed intensity variations of the spectral features. The asymmetric displacement of the central Coulomb gap and of the $C_{2}$-related peaks under the action of an external voltage pulse reflects the different values of the fractional residual charge $Q_{0}$, obtained from our simulation, on the $\mathrm{Pb}$ island.

It has been proposed that the residual charge $Q_{0}$ is related to the difference in work function of the various metals constituting the junctions (12):

$$
Q_{0}=\left[C_{2}\left(\Delta \phi_{2}\right)-C_{1}\left(\Delta \phi_{1}\right)\right] / e
$$

where $\Delta \phi_{1}$ is the difference between substrate and island work functions, and $\Delta \phi_{2}$ the difference between tip and island work functions. As a result of voltage pulses, charge can be trapped in defects or impurities in the $\mathrm{NaCl}$ layer beneath the $\mathrm{Pb}$ nanoisland, leading to a permanent modification of the residual charge $Q_{0}$.

In the following section we demonstrate the influence of the tip-island junction on the residual charge $Q_{0}$ via the variation of $C_{2}$, variation due to the displacement of the tip with respect to the $\mathrm{Pb}$ island during scanning. In Figures 7A,B two different $\mathrm{Pb}$ islands are displayed. In the closeup-view topographic images of Figures 7C,D, very striking concentric lines following the contours of the island are visible. Figures 7E,F shows typical $d I / d V$ spectra obtained on these islands. Our measurements show that, by scanning laterally across the surface, an energy shift of the spectrum is observed which corresponds to a maximum fluctuation of the residual charge $Q_{0}= \pm e / 2$. We deduced the $Q_{0}$ values by simulating each local STS spectrum in the $\mathrm{CS}$ regime. For the $\mathrm{Pb}$ island shown in Figures 7A,C, we obtain a value of $Q_{0} \approx+0.35 e$ for the spectrum acquired at the center of the island, and $Q_{0} \approx-0.15 e$ for the one acquired in the surrounding region. For the system of Figures 7B,D, up to six regions of alternating negative and positive $Q_{0}$ are detected.

In order to explain this surprising effect we have to consider that the parameters of junction 2 depend on the tip position with respect to the island. As already mentioned, the residual charge $Q_{0}$ is related to the difference in work function of the various metals constituting the junctions, see Equation 1. For a given set of measurements $C_{1}$ is fixed by the $\mathrm{Pb}$ island and the $\mathrm{NaCl}$ layer characteristics and the work functions are well defined. In these conditions, Equation 1 shows that a variation of $Q_{0}$ can only originate from a variation of $C_{2}$. As discussed above, the lateral extension of the tip can exceed the island area. Therefore, to evaluate $C_{2}$, not only the capacitance between tip and island, but also the one between tip and substrate has to be included. When scanning over a $\mathrm{Pb}$ island, the ratio between these two contributions changes continuously, leading to variations of the residual charge within the island. Now, considering the CS spectra shown in Figures 7E,F, we recall that with increasing bias voltage every additional CS peak in the differential conductance spectrum indicates the hopping of an additional electron to/from the island. In an STM scan at a bias voltage close to a CS peak, e.g., at $0.075 \mathrm{eV}$ in Figure 7E, a small change in $Q_{0}$ (due to the changing tip-island capacitance) will lead to a situation where the measurement will be performed above or below the respective CS peak, corresponding to one more (above the peak) or one less electron (below the peak) tunneling to the island. This effect leads to the observed symmetric triangular step patterns in the STM 

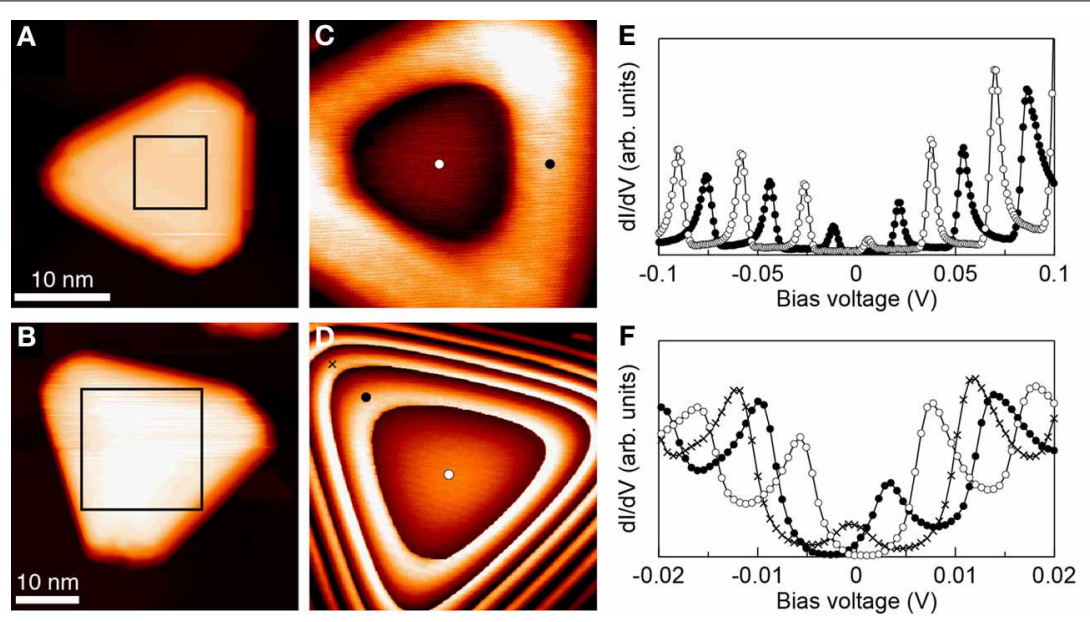

FIGURE 7 | (A,B) Topographic images of Pb islands on NaCl; (A): -1 V, 20 pA, (B): 2.5 V, 20 pA; (C,D) Close-up view; (C): -0.15V, 200 pA; (D): -0.01 V, 20 pA); (E,F) $d l / d V$ spectra acquired on the locations represented by the corresponding symbol, showing different values of $Q_{0}$ (see text).

topographies shown in Figures 7A,B. Evidently, the sharp apparent height steps visible in the STM images reflect the hopping of one additional electron on/off the $\mathrm{Pb}$ island, while the transition regions correspond to a continuous variation of $Q_{0}$. Notice that, contrary to the first impression gained from the images, at each instant the value of the charge $Q_{0}$ is the same over the entire island, as expected for a conducting object. These features are visible when $C_{1} \Delta \phi_{1} \approx C_{2} \Delta \phi_{2}$, a condition which can be fulfilled for $\Delta \phi_{1}$ ten times smaller than $\Delta \phi_{2}$, since $C_{1}$ is typically ten times larger than $C_{2}$. This implies that $\Delta \phi_{1}$ has to be small in order to observe these features. Moreover, $\Delta \phi_{2}$ strongly depends on the specific tip conditions, explaining the fact that these effects were not systematically observed. In this situation, a $C_{2}$ variation of the order of $10 \%$ can induce a modification of $Q_{0}$ as large as $\pm e / 2$.

Finally, we note that the observation of Coulomb charge rings with scanning probe methods has been made before for quantum dots inside carbon nanotubes (50), for quantum dots of a two-dimensional electron gas inside semiconductor heterostructures (51), and for quantum dots inside InAs nanowires (52). Controlled charge switching has been performed for single $\mathrm{Au}$ atoms on $\mathrm{NaCl}$ (53), for a single $\mathrm{Si}$ donor in Si doped GaAs (54), and for a small conductive grain on an InAs surface (55). The present measurements on $\mathrm{Pb}$ nanoislands show that these intriguing $\mathrm{CB}$ phenomena and the related charging effects are also observed in metallic quantum dots. While the observed phenomena are similar they are not identical. The essential difference between the present measurements and the SPM investigations on the quantum dots before is that in our case the STM tip is in the tunneling mode measuring the conductance through the $\mathrm{Pb}$ island without a gate electrode, while the former investigations use a standard conductance measurement of the nanowires (quantum dots) and there the SPM tip represents an external electrode the field of which shifts the CS spectra of the observed quantum dots.

\section{CONCLUSIONS AND OUTLOOK}

We investigated the transport properties of single crystalline metallic nanostructures in the $\mathrm{CB}$ regime. To this end, small $\mathrm{Pb}$ islands with well-defined geometries, ranging from a diameter of a few to tens of nanometers and a thickness of a few monolayers are grown on crystalline $\mathrm{NaCl}$ layers on $\mathrm{Ag}(111)$ substrate. The tunneling spectra obtained at $4.6 \mathrm{~K}$ on the metallic quantum dots show clearly the presence of CB phenomena. By reducing the tipisland distance, the tunneling spectra reflect the evolution from CB to CS. Our simulations employing the semi-classical orthodox theory describe well the observed spectra in both regimes allowing us to extract quantitatively the parameters of the DBTJ, i.e., the resistances, capacitances, and the residual charges.

The present STM/STS studies have shown that not only the size and shape of the metallic nanoislands can be well determined but that, importantly, also their local electronic structure and their transport properties can be quantitatively elucidated. In the past, e.g., the superconducting properties of small particles have been investigated (56) revealing a number parity property. STS experiments of isolated superconducting islands would allow us to observe simultaneously the pairing correlation on islands with different parity. Moreover, in small metallic particles containing magnetic impurities, the Kondo resonance was predicted to be strongly affected in a parity-dependent way (4), another exciting phenomenon to look at with local probes. We believe that STM/STS will continue to be a decisive player for the elucidation of the mesoscopic physics of nanostructures.

\section{ACKNOWLEDGMENTS}

We would like to thank K.-H. Müller and C. Flindt for stimulating discussions. We are also indebted to V. S. Stepanyuk, N. N. Negulyaev, P. Ignatiev, K. Horn and D. Roditchev for their very valuable input. Wolf-Dieter Schneider would like to thank H.-J. Freund for stimulating discussions and for his generous hospitality. We acknowledge financial support of the Swiss National Science Foundation. 


\section{REFERENCES}

1. Sohn LL, Kouwenhoven LP, Schön G. Mesoscopic electron transport. Dordrecht: Kluwer Academic Publishers; (1997).

2. Grabert H, Devoret MH, (eds). Single Charge Tunneling. Vol. 294 of NATO ASI Series B: Physics. New York: Plenum Press (1992).

3. Likharev KK. Single-electron devices and their applications. Proc IEEE. (1999) 87:606. doi: $10.1109 / 5.752518$

4. von Delft J, Ralph DC. Spectroscopy of discrete energy levels in ultrasmall metallic grains. Phys Rep. (2001) 345:61. doi: 10.1016/S0370-1573(00)00099-5

5. Aleiner IL, Brouwer PW, Glazman LI. Quantum effects in Coulomb blockade. Phys Rep. (2002) 358:309. doi: 10.1016/S0370-1573(01)00063-1

6. Zeller HR, Giaever I. Tunneling, zero-bias anomalies, and small superconductors. Phys Rev. (1969) 181:789. doi: 10.1103/PhysRev.181.789

7. Lambe J, Jaklevic RC. Chargequantization studies using a tunnel capacitor. Phys Rev Lett. (1969) 22:1371. doi: 10.1103/PhysRevLett.22.1371

8. Fulton TA, Dolan GJ. Observation of single-electron charging effects in small tunneljunctions. Phys Rev Lett. (1987) 59:109. 10.1103/ PhysRevLett.59.109

9. Iansiti M, Johnson AT, Lobb CJ, Tinkham M. Crossover from Josephson Tunneling to the Coulomb Blockade in Small Tunnel Junctions. Phys Rev Lett. (1988) 60:2414. doi: 10.1103/PhysRevLett.60.2414

10. van Bentum PJM, Smokers RTM, van Kempen $H$. Incremental Charging of Single Small Particles. Phys Rev Lett. (1988) 60:2543. doi: 10.1103/ PhysRevLett.60.2543

11. Wilkins R, Benjacob E, Jaklevic RC. Scanning-tunnelingmicroscope observations of Coulomb blockade and oxide-polarization in small metal droplets. Phys Rev Lett. (1989) 63:801. doi: 10.1103/PhysRevLett.63.801

12. Hanna AE, Tinkham M. Variation of the Coulomb staircase in a 2junction system by fractional electron charge. Phys Rev B. (1991) 44:5919. doi: 10.1103/PhysRevB. 44.5919

13. Amman M, Field SB, Jaklevic RC. Coulomb -blockade spectrocopy of gold particles imaged with scanning-tunneling-microscopy. Phys Rev B. (1993) 48:12104. doi: 10.1103/PhysRevB.48.12104

14. Kulik IO, Shekhter RI. Kinetic phenomena and charge discreteness effects in granulated media. Sov Phys JETP. (1975) 41:308.

15. Averin DV, Likharev KK. Coulomb Blockade of SingleElectron Tunneling, and Coherent Oscillations in Small Tunnel Junctions. J Low Temp Phys. (1986) 62:345. doi: 10.1007/BF00683469

16. Averin DV, Likharev KK. Mesoscopic Phenomena in Solids. vol. 30 of Modern Problems in Condensed Matter Sciences. Altshuler BL, Lee PA, Webb RA, (eds). Amsterdam: North-Holland; (1991).

17. Oncel N, Hallback AS, Zandvliet HJW, Speets EA, Ravoo BJ, Reinhoudt DN, et al. Coulomb blockade of small Pd clusters. J Chem Phys. (2005) 123:044703. doi: 10.1063/1.1996567

18. Koslowski B, Dietrich C, Weigl F, Ziemann P. Dynamic charging at room temperature of $\mathrm{Au}$ nanoparticles prepared by a micellar technique. Phys Rev B. (2007) 75:085407. doi: 10.1103/PhysRevB.75.085407

19. Dubois JGA, Gerritsen JW, Shafranjuk SE, Boon EJG, Schmid $\mathrm{G}$, et al. Coulomb staircases and quantum size effects in tunnelling spectroscopy on ligand-stabilized metal clusters. Europhys Lett. (1996) 33:279. doi: 10.1209/epl/i1996-00333-0

20. Banin U, Cao YW, Katz D, Millo O. Identification of atomiclike electronic states in indium arsenide nanocrystal quantum dots. Nature (1999) 400:542. doi: $10.1038 / 22979$

21. Wang $\mathrm{B}$, Wang $\mathrm{H}$, Li $\mathrm{H}$, Zeng C, Hou JG, Xiao X. Tunable single-electron tunneling behavior of ligand-stabilized gold particles on self-assembled monolayers. Phys Rev B. (2000) 63:035403. doi: 10.1103/ PhysRevB.63.035403

22. Wang B, Wang KD, Lu W, Yang JL, Hou JG. Size-dependent tunneling differential conductance spectra of crystalline Pd nanoparticles. Phys Rev B. (2004) 70:205411. doi: 10.1103/PhysRevB.70.205411

23. Andres RP, Bein T, Dorogi $M$, Feng S, Henderson JI, Kubiak CP, et al. Coulomb staircase" at room temperature in a self-assembled molecular nanostructure. Science (1996) 272:1323.
24. Nilius N, Kulawik M, Rust HP, Freund HJ. Erratum to: quantization of electronic states in individual oxide-supported silver particles [Surface Science 572 (2004) 347]. Surf Sci. (2006) 600:3522. doi: 10.1016/j.susc.2006.06.022

25. Schouteden K, Vandamme $\mathrm{N}$, Janssens E, Lievens $\mathrm{P}$, Van Haesendonck C. Singleelectron tunneling phenomena on preformed gold clusters deposited on dithiol self-assembled monolayers. Surf Sci. (2008) 602:552. doi: 10.1016/j.susc.2007.11.006

26. Pauly C, Grob M, Pezzotta M, Pratzer M, Morgenstern M. Gundlach oscillations and Coulomb blockade of $\mathrm{Co}$ nanoislands on $\mathrm{MgO} / \mathrm{Mo}(100$ investigated by scanning tunneling spectroscopy at $300 \mathrm{~K}$. Phys Rev B. (2010) 81:125446. doi: 10.1103/PhysRevB.81.125446

27. Brun C, Müller $\mathrm{KH}$, Hong IP, Patthey F, Flindt C, Schneider WD. Dynamical coulomb blockade observed in nanosized electrical contacts. Phys Rev Lett. (2012) 108:126802. doi 10.1103/PhysRevLett.108.126802

28. Devoret MH, Esteve D, Grabert $\mathrm{H}$, Ingold GL, Pothier H, Urbina C. Effect of the electromagnetic environment on the coulomb blockade in ultrasmall tunnel-junctions. Phys Rev Lett. (1990) 64:1824. doi: 10.1103/PhysRevLett.64.1824

29. Girvin SM, Glazman LI, Jonson M, Penn DR, Stiles MD. Quantum fluctuations and the single-junction Coulomb blockade. Phys Rev Lett. (1990) 64:3183. doi: 10.1103/ PhysRevLett.64.3183

30. Ingold GL, Nazarov YV. Single Charge Tunneling. New York: Plenum (1992).

31. Gaisch R, Gimzewski JK, Reihl B, Schlittler RR, Tschudy M, Schneider WD. Low-temperature ultra-highvacuum scanning tunneling microscope. Ultramicroscopy. (1992) 42:1621. doi: 10.1016/0304-3991(92)90495-6

32. Goldmann A, Koch EE. Condensed Matter. vol. $23 a$ of LandoltBornstein - Group III Condensed Matter. Berlin; Heidelberg; New York; London; Paris; Tokyo; Hongkong: $\quad$ Springer-Verlag (1989).

33. Haensel R, Keitel G, Peters G, P S, Sonntag B, Kunz C. Photoemission measurement on $\mathrm{NaCl}$ in photon energy range 32-50 eV. Phys
Rev Lett. (1969) 23:530. doi: 10.1103/PhysRevLett.23.530

34. Poole RT, Jenkin JG, Liesegang J, Leckey RCG. Electronic bandstructure of Alkali-Halides .1. Experimental parameters. Phys Rev B. (1975) 11:5179. doi: 10.1103/PhysRevB.11.5179

35. Hebenstreit W, Redinger J, Horozova Z, Schmid M, Podloucky R, Varga P. Atomic resolution by STM on ultra-thin films of alkali halides: experiment and local density calculations. Surf Sci. (1999) 424:L321. doi: $\quad 10.1016 / S 0039-6028(99)$ 00095-3

36. Ploigt HC, Brun C, Pivetta M, Patthey F, Schneider WD. Local work function changes determined by field emission resonances: $\mathrm{NaCl} / \mathrm{Ag}(100)$. Phys Rev B. (2007) 76:195404. doi: 10.1103/PhysRevB.76.195404

37. Barjenbruch U, Fölsch S, Henzler M. Surface-states on epitaxial thin-films of $\mathrm{NaCl}$ and $\mathrm{KCl}$. Surf Sci. (1989) 211:749. doi: 10.1016/0039-6028(89)90837-6

38. Hebenstreit W, Schmid M, Redinger J, Podloucky R, Varga P. Bulk terminated $\mathrm{NaCl}(111)$ on aluminum: a polar surface of an ionic crystal? Phys Rev Lett. (2000) 85:5376. doi: 10.1103/PhysRevLett.85.5376

39. Repp J, Meyer G, Stojković SM, Gourdon A, Joachim C. Molecules on insulating films: scanning-tunneling microscopy imaging of individual molecular orbitals. Phys Rev Lett. (2005) 94:026803.

40. Kramer J, Tegenkamp C, Pfnür H. The growth of $\mathrm{NaCl}$ on flat and stepped silver surfaces. J Phys-Condens Matter. (2003) 15:6473. doi: 10.1088/0953-8984/15/38/015

41. Pivetta M, Patthey F, Stengel M, Baldereschi A, Schneider WD. Local work function Moiré pattern on ultrathin ionic films: $\mathrm{NaCl}$ on $\mathrm{Ag}(100)$. Phys Rev B. (2005) 72: 115404.

42. Ćavar E, Blüm MC, Pivetta M, Patthey F, Chergui M, Schneider WD. Fluorescence and phosphorescence from individual C-60 molecules excited by local electron tunneling. Phys Rev Lett. (2005) 95:196102.

43. Rossel F, Pivetta M, Patthey F, Schneider WD. Plasmon enhanced luminescencefrom fullerene molecules excited bylocal electron tunneling. Opt Express. (2009) 17:2714. doi: 10.1364/OE.17.002714 
44. Cañas Ventura ME, Xiao W, Ruffieux P, Rieger R, Müllen K, et al. Stabilization of bimolecular islands on ultrathin $\mathrm{NaCl}$ films by a vicinal substrate. Surf Sci. (2009) 603:2294.

45. Brun C, Hong IP, Patthey F, Sklyadneva IY, Heid R, Echenique $\mathrm{PM}$, et al. Reduction of the superconducting gap of ultrathin $\mathrm{Pb}$ islands grown on $\mathrm{Si}(111)$. Phys Rev Lett. (2009) 102:207002. doi: 10.1103/PhysRevLett.102.207002

46. Robinson MC, Hallett ACH. Static dielectric constant of $\mathrm{NaCl}, \mathrm{KCl}$, and $\mathrm{KBr}$ at temperature between 4.2 degrees $\mathrm{K}$ and 300 degrees $\mathrm{K}$. Can J Phys. (1966) 44:2211. doi: 10.1139/p66-179

47. Hong IP, Brun C, Patthey F, Sklyadneva IY, Zubizarreta X, Heid R, et al. Decay mechanisms of excited electrons in quantumwell states of ultrathin $\mathrm{Pb}$ islands grown on $\mathrm{Si}(111)$ : scanning tunneling spectroscopy and theory. Phys Rev B. (2009) 80:081409. doi: 10.1103/PhysRevB.80.081409

48. Banin U, Millo O. Tunneling and optical spectroscopy of semi- conductor nanocrystals. Annu Rev Phys Chem. (2003) 54:465. doi: 10.1146/annurev.physchem. 54.011002 .103838

49. Ralph DC, Black CT, Tinkham M. Gate-voltage studies of discrete electronic states in aluminum nanoparticles. Phys Rev Lett. (1997) 78:4087. doi: 10.1103/PhysRevLett.78.4087

50. Woodside MT, McEuen PI. Scanned probe imaging of single-electron charge states in nanotube quantum dots. Science (2002) 296:1098. doi: 10.1126/science. 1069923

51. Fallahi P, Bleszynski AC, Westervelt RM, Huang J, Walls JD, Heller EJ, et al. Imaging a single-electron quantum dot. Nano Lett. (2005) 5:223. doi: $10.1021 / \mathrm{nl} 048405 \mathrm{v}$

52. Bleszynski AC, Zwanenburg FA, Westervelt RM, Roest AL, Bakkers EPAM, et al. Scanned probe imaging of quantum dots inside InAs nanowires. Nano Lett. (2007) 7:2559.

53. Repp J, Meyer G, Olsson FE, Persson M. Controlling the charge state of individual gold atoms. Science. (2004) 305:493. doi: 10.1126/science.1099557

54. Teichmann K, Wenderoth $\mathrm{M}$, Loth S, Ulbrich RG, Garleff JK, Wijnheijmer AP, et al. IControlled charge switching on a single donor with a scanning tunneling microscope. Phys Rev Lett. (2008) 101:076103. doi: 10.1103/PhysRevLett.101.076103

55. Wildoer JWG, van Roij AJA, Harmans CJPM, van Kempen H. Semiconductor band switching by charging a small grain with a single electron. Phys Rev B. (1996) 53:10695. doi: 10.1103/PhysRevB.53.10695

56. Black CT, Ralph DC, Tinkham M. Spectroscopy of the superconducting gap in individual nanometer-scale aluminum particles. Phys Rev Lett. (1996) 76:688. doi: 10.1103/PhysRevLett. 76.688

Conflict of Interest Statement: The authors declare that the research was conducted in the absence of any commercial or financial relationships that could be construed as a potential conflict of interest.

Received: 23 July 2013; paper pending published: 11 August 2013; accepted: 06 September 2013; published online: 27 September 2013.

Citation: Hong I-P, Brun C, Pivetta $M$, Patthey $F$ and Schneider W-D (2013) Coulomb blockade phenomena observed in supported metallic nanoislands. Front. Physics 1:13. doi: 10.3389/fphy.2013.00013

This article was submitted to Condensed Matter Physics, a section of the journal Frontiers in Physics.

Copyright (C) 2013 Hong, Brun, Pivetta, Patthey and Schneider. This is an open-access article distributed under the terms of the Creative Commons Attribution License (CC BY). The use, distribution or reproduction in other forums is permitted, provided the original author(s) or licensor are credited and that the original publication in this journal is cited, in accordance with accepted academic practice. No use, distribution or reproduction is permitted which does not comply with these terms. 